\title{
The Uncertainty of Storm Season Changes: Quantifying the Uncertainty of Autocovariance Changepoints
}

\author{
Christopher F. H. Nam ${ }^{1}$, John A. D. Aston ${ }^{1,2}$, Idris A. Eckley ${ }^{3}$, Rebecca Killick ${ }^{3}$ \\ ${ }^{1}$ Department of Statistics, University of Warwick, Coventry, CV4 7AL, UK \\ ${ }^{2}$ Statistical Laboratory, DPMMS, University of Cambridge, Cambridge, CB3 0WB, UK \\ ${ }^{3}$ Department of Mathematics and Statistics, University of Lancaster, Lancaster, LA1 4YF, UK \\ \{c.f.h.nam j.a.d.aston \}@warwick.ac.uk \\ \{i.eckley | r.killick \}@lancaster.ac.uk
}

February 13, 2014

\begin{abstract}
In oceanography, there is interest in determining storm season changes for logistical reasons such as equipment maintenance scheduling. In particular, there is interest in capturing the uncertainty associated with these changes in terms of the number and location of them. Such changes are associated with autocovariance changes. This paper proposes a framework to quantify the uncertainty of autocovariance changepoints in time series motivated by this oceanographic application. More specifically, the framework considers time series under the Locally Stationary Wavelet framework, deriving a joint density for scale processes in the raw wavelet periodogram. By embedding this density within a Hidden Markov Model framework, we consider changepoint characteristics under this multiscale setting. Such a methodology allows us to model changepoints and their uncertainty for a wide range of models, including piecewise second-order stationary processes, for example piecewise Moving Average processes.
\end{abstract}

Keywords: Changepoints; Hidden Markov Models; Locally Stationary Wavelet processes; Oceanography; Sequential Monte Carlo. 


\section{Introduction}

In oceanography, historic wave height data is often used to determine storm season changes. Identifying such changes in season provides a better understanding of the data for oceanographers which may help them in planning future maintenance work of equipment such as offshore oil rigs. Changes in autocovariance structure are associated with these storm season changes and thus variance (Killick et al., 2009) and autocovariance changepoint methods (Killick et al., 2013) are employed in determining these changes. However, there is often uncertainty and ambiguity associated with these changes, such as their number and location, which traditional changepoint methods often fail to capture. This paper thus proposes a methodology in which changes in autocovariance structure are considered and the uncertainty associated with such changes is captured explicitly.

Work in changepoint detection and estimation has focused on detecting changes in mean, trend (regression), variance, and combinations thereof; see Chen and Gupta (2000) and Eckley et al. (2011) for overviews. However, changes in autocovariance can also occur, although there is comparatively little changepoint literature dedicated to such changes. In addition, different changepoint methods often provide different changepoint estimates, for example the number and location of changepoints, and many fail to capture explicitly the uncertainty of these estimates. As Nam et al. (2012b) argue, there is consequently a need to assess the plausibility of estimates provided by different changepoint methods in general. Quantifying the uncertainty of changepoints provides one method of doing so for this autocovariance setting.

Methods for detecting and estimating changes in autocovariance have recently been proposed. Davis et al. (2006) propose the Automatic Piecewise Autoregressive Modelling (AutoPARM) procedure which models observed time series as piecewise AR processes with varying orders and AR coefficients. Changepoints are identified via optimisation of the minimum description length criteria which provides the best segmentation configuration. However the assumption of piecewise AR processes is a strong assumption and may not always be appropriate. Uncertainty is implicitly captured via asymptotic arguments in obtaining consistent estimates of the changepoint locations, conditional on the number of changepoints being known, and thus not reported explicitly.

Cho and Fryzlewicz (2012) consider identifying changepoints in periodograms of the time 
series. Periodograms describe the autocovariance structure of a time series in the frequency domain. Specifically, Cho and Fryzlewicz (2012) consider modelling time series under the Locally Stationary Wavelet (LSW) framework. Under this framework, the Evolutionary Wavelet Spectrum (EWS) describes the autocovariance structure of a time series at different scales (frequency bands) and locations. Autocovariance changepoints in the time series thus correspond to changes in the scale processes of the EWS and vice versa. Changepoint analysis now focuses on identifying changepoints in these scale processes. Cho and Fryzlewicz (2012) (CF) analyse each scale process independently for changepoints via a non-parametric test statistic, and then combine changepoint results from each scale to obtain a single set of results for the observed time series. The non-parametric test statistic places less restriction on the time series considered although several tuning parameters are required under this approach and so care is required. In general, the wavelet-domain and the LSW framework potentially allow new types of models and data to be considered which are not feasible in the time-domain. This includes moving average processes.

Figure 1 illustrates the phenomenon of obtaining different changepoint results when the aforementioned changepoint methods are applied to the wave height data. This paper consequently attempts to address this discrepancy in changepoint results by providing a methodology which explicitly yields the uncertainty of any autocovariance (second-order) changes provided. If uncertainty regarding changepoints is given, then it is possible to decide as to whether a time point that is deemed a changepoint is almost certain to be so, or whether a changepoint is likely to have occurred within an interval, but the exact location within the interval is much less certain.

[Figure 1 about here.]

Building upon the existing wavelet-based approach of Cho and Fryzlewicz (2012), we model the time series as a LSW process and perform our analysis using the wavelet periodogram, an estimate of the EWS. We derive a joint density for scale processes of the raw wavelet periodogram which can be embedded into a Hidden Markov Model (HMM) framework, a popular framework to model non-linear and non-stationary time series. This HMM framework allows a variety of existing changepoint methods to potentially be applied (for example changes in state in the Viterbi sequence (Viterbi, 1967)), with our focus being that of quantifying the uncertainty of 
changepoints as proposed in Nam et al. (2012b).

Time-domain HMMs are currently unable to model some time series with changing autocovariance structures without some approximation taking place. This includes piecewise moving average processes. Our work thus proposes a wavelet based HMM framework such that time series exhibiting piecewise autocovariance structures can be considered more actively. As such, this framework allows us to quantify explicitly the uncertainty of second-order structure changepoints, an aspect which is not considered in existing changepoint methods.

The structure of this paper is as follows: Section 2 reviews the statistical background that the proposed methodology is built upon. Section 3 explains the proposed framework and modelling approach. Section 4 applies the proposed framework to a variety of simulated data and the oceanographic data as presented in Figure 1. Section 5 concludes the paper.

\section{Statistical Background}

Let $y_{1}, \ldots, y_{n}$ denote a potential non-stationary time series, observed at equally spaced discrete time points. We assume that the non-stationarity arises due to a varying second-order structure such that for any lag $v \geq 0$, there exists a $\tau$ such that

$$
\operatorname{Cov}\left(Y_{1}, Y_{v}\right)=\ldots=\operatorname{Cov}\left(Y_{\tau-1}, Y_{\tau-v}\right) \neq \operatorname{Cov}\left(Y_{\tau}, Y_{\tau-v+1}\right)=\ldots=\operatorname{Cov}\left(Y_{n-v+1}, Y_{n}\right)
$$

and that the mean remains constant. In situations where the mean is not constant, preprocessing of the data can be performed. We refer to $\tau$ as a changepoint. Changes in secondorder structure can be constructed easily; for example by a piecewise autoregressive moving average (ARMA) process.

One approach in modelling time series exhibiting non-stationarity such as changes in mean and variance is via Hidden Markov Models (HMMs). For overviews of HMMs, we refer the reader to MacDonald and Zucchini (1997) and Cappé et al. (2005). The use of HMMs provides a sophisticated modelling framework for a variety of problems and applications including changepoint analysis (for example Chib (1998), Aston et al. (2011)) and thus forms one of the many building blocks in our proposed methodology. Within a HMM framework, we have the observation process $\left\{Y_{t}\right\}_{t \geq 1}$ which is dependent on an underlying latent finite state Markov chain (MC), $\left\{X_{t}\right\}_{t \geq 0} \in \Omega_{X}$ with $\left|\Omega_{X}\right|<\infty$. The states of the underlying MC can repre- 
sent different data generating mechanisms, for example, "stormy" and "non-stormy" seasons in the oceanographic application we are interested in. Under the HMM framework, the observations, $Y_{1: n} \equiv\left(Y_{1}, \ldots, Y_{n}\right)$ are conditionally independent given the underlying state sequence $X_{0: n} \equiv\left(X_{0}, \ldots, X_{n}\right)$.

However as Frühwirth-Schantter (2005) observes, a time-domain based HMM framework is not suitable for directly modelling time series data arising from piecewise ARMA models as the entire underlying state sequence needs to be recorded for inference. In order to apply the desired HMM framework for such data, an alternative approach is required. In this paper, we investigate the potential of transforming the problem to an alternative domain, namely the wavelet domain.

\section{$2.1 \quad$ Locally Stationary Wavelet Processes}

Wavelets are compactly supported oscillating functions which permit a time series or function to be equivalently represented at different scales (frequency bands) and locations. We refer interested readers to Vidakovic (1999), Percival and Walden (2007) and Nason (2008) for comprehensive overviews of wavelets in statistics and time series analysis. Due to the time localisation properties of wavelets, they are a natural method to use when considering changepoints and discontinuities in time series. Hence, in recent years, they have been used to detect changes in means (Wang, 1995), changes in variance (Whitcher et al., 2000) and changes in autocorrelation structure (Choi et al., 2008).

The Locally Stationary Wavelet (LSW) framework is a popular wavelet based modelling framework for non-stationary time series arising from a varying second-order structure, including all piecewise second order stationary processes (Nason et al., 2000), which has already seen application in changepoint detection (but without associated quantification) in Cho and Fryzlewicz (2012) and Killick et al. (2013). Following Fryzlewicz and Nason (2006), we adopt the following definition of a LSW process,

Definition 1. $\left\{Y_{t}\right\}_{t=1}^{n}$ for $n=1,2, \ldots$, is said to be a Locally Stationary Wavelet (LSW) process if the following mean-square representation exists,

$$
Y_{t}=\sum_{j=1}^{\infty} \sum_{k \in \mathbb{Z}} \psi_{j, k}(t) W_{j}\left(\frac{k}{n}\right) \xi_{j, k}
$$


where $j \in \mathbb{N}$ and $k \in \mathbb{Z}$ denote the scale and location parameters respectively. $\psi_{j}=\left\{\psi_{j, k}\right\}_{k \in \mathbb{Z}}$ is a discrete, real-valued, compactly supported, non-decimated wavelet vector with support lengths $\mathcal{L}_{j}=\mathcal{O}\left(2^{j}\right)$ at each scale, with $\psi_{j, k}(t)=\psi_{j, k-t}$, the wavelet shifted by $t$ positions. $\xi_{j, k}$ is a zero-mean, orthonormal, identically distributed incremental error process.

For each $j \geq 1, W_{j}(z):[0,1] \rightarrow \mathbb{R}$ is a real valued, piecewise constant function with a finite (but unknown) number of jumps. Let $\mathcal{N}_{j}$ denote the total magnitude of the jumps in $W_{j}^{2}(z)$, the variability of function $W_{j}^{2}(z)$ is controlled so that

- $\sum_{j=1}^{\infty} W_{j}(z)<\infty$ uniformly in $z$.

- $\sum_{j=1}^{J} 2^{j} \mathcal{N}_{j}=\mathcal{O}(\log n)$ where $J=\left\lfloor\log _{2} n\right\rfloor$.

By definition, an LSW process assumes $Y_{t}$ has mean zero for all $t$. The motivation for the LSW framework is that observed time series are often non-stationary over the entire observed time period (globally), but may be stationary if we analyse them in shorter time windows (locally). Analogous to classical Fourier time series analysis, the Evolutionary Wavelet Spectrum (EWS), $W_{j}^{2}\left(\frac{k}{n}\right), j=1,2, \ldots$, characterises the second-order structure of the LSW process, $Y_{t}$, up to the choice of wavelet basis. Note in particular that under this definition, the EWS is piecewise constant.

Given an observed time series $\left\{Y_{t}\right\}_{t=1}^{n}$, an estimate of the EWS can be obtained by considering the square of the empirical wavelet coefficients from a non-decimated wavelet transform (NDWT) of the series. That is,

$$
W_{j}^{2}\left(\frac{k}{n}\right) \approx I_{j, k}=D_{j, k}^{2}=\left(\sum_{t=1}^{n} \psi_{j, k}(t) Y_{t}\right)^{2}
$$

This is referred to as the raw wavelet periodogram in the literature. Nason et al. (2000) established that this raw form of the wavelet peridogram is a biased estimator of the EWS, the (asymptotic) form of the bias being described by the inner product matrix of discrete autocorrelation wavelets. Whilst in general it may be preferable to use an (asymptotically) unbiased form of the periodogram for general inference, within the changepoint context which we consider, Cho and Fryzlewicz (2012) establish that every breakpoint in the autocovariance structure results in a breakpoint in the (raw) periodogram. Hence, for the purposes of this article, it suffices to focus our inference based on this (biased) periodogram. 
For a sequence of random variables, $Y_{1: n}$, we denote the corresponding raw wavelet periodogram as $\mathbf{I}_{1: n}$. This is a multivariate time series consisting of $J=\left\lfloor\log _{2} n\right\rfloor$ components at each location, with each component denoting a different scale. $\mathbf{i}_{1: n}=\mathbf{d}_{1: n}^{2}$ denotes the empirical raw wavelet periodogram corresponding to observed time series $y_{1: n}$.

Both the HMM and LSW frameworks are powerful tools in modelling different types of nonstationary time series. A natural question to thus ask is whether it is possible to combine the two frameworks such that HMM-based changepoint methods can be applied within the LSW framework. The hybrid framework would thus allow us to consider changes in second-order structure through the LSW framework, whilst applying a multitude of existing HMM-based changepoint methods (for example Chib (1998), Aston et al. (2011) and Nam et al. (2012b)). Section 3 proposes a framework in which this can be achieved.

\section{Methodology}

As previously described, our goal is to quantify the uncertainty of autocovariance changepoints for a time series by considering its spectral structure. Quantities of interest include the changepoint probability $P\left(\tau=t \mid y_{1: n}\right)$ (CPP, the probability of a changepoint at time $\left.t\right)$, and the distribution of number of changepoints within the observed time series $P\left(N_{C P}=n_{C P} \mid y_{1: n}\right)$. Other changepoint characteristics such as joint or conditional changepoint distributions are also available using the proposed methodology.

The raw wavelet periodogram characterises how the second-order structure evolves over time. Thus, we perform analysis on the periodogram to quantify the uncertainty of secondorder changepoints. This is achieved by modelling the periodogram via a HMM framework, and quantifying the changepoint uncertainty via an existing HMM approach (Nam et al., 2012b). In proposing the new methodology, several challenges need to be addressed.

Firstly, the multivariate joint density of $\mathbf{I}_{k}$ is unknown and needs to be derived. This density also captures the dependence structure introduced by the use of the NDWT in estimating the periodogram. The derivation of this joint density and its embedding in a HMM modelling framework is detailed in Section 3.1. As the model parameters, $\theta$, associated with the HMM framework are unknown, these need to be estimated and we turn to Sequential Monte Carlo samplers (SMC, Del Moral et al. (2006)) in considering the posterior of the parameters. These 
model parameters can be shown to be directly associated with the EWS. An example SMC implementation is provided in Section 3.2. Section 3.3 details some aspects concerning the computation of the distribution of changepoint characteristics. Section 3.4 provides an outline of the overall proposed approach.

There are many advantages to considering the observed time series under the LSW framework. In particular, time series exhibiting piecewise second-order structure can be more readily analysed under this framework compared to a time-domain approach. For example, for a piecewise moving average process, the associated EWS has a piecewise constant structure at each scale; a sparser representation where the discontinuities can be analysed with fewer issues potentially arising. This sparser representation is not possible in the time-domain and is thus one of the attractions of the LSW framework.

By combining the use of wavelets in conjunction with a HMM framework, we can systematically induce a dependence structure in the HMM framework compared to choosing an arbitrary dependence structure in the time-domain.

We assume in this paper that the error process in the LSW model is Gaussian, that is $\xi_{j, k} \stackrel{\text { iid }}{\sim} \mathrm{N}(0,1)$. This leads to $Y_{t}$ being Gaussian itself and is commonly referred to as a Gaussian LSW process. Recall from Section 2.1 that our EWS is piecewise constant. That is,

$$
W_{j}^{2}\left(\frac{k}{n}\right)=\sum_{r=1}^{H^{*}} w_{j, r}^{2} \mathbf{1}_{\mathcal{W}_{r}}(k) \quad j=1, \ldots, J,
$$

where $w_{j, r}^{2}$ are some unknown constants, and $\mathcal{W}_{r}, r=1, \ldots, H^{*}$ is an unknown disjoint partitioning of $1, \ldots, n$ over all scales $j$ simultaneously. Each $\mathcal{W}_{r}$ has a particular EWS power structure associated with it, such that consecutive $\mathcal{W}_{r}$ have changes in power in at least one scale. $H^{*}$ denotes the unknown number of partitions there are in the EWS (and hence also in the raw periodogram), and ultimately corresponds to the segments in the data and in turn the number of changepoints.

We now propose the LSW-HMM modelling framework in quantifying the uncertainty of autocovariance changepoints under the assumptions outlined above.

\subsection{LSW-HMM modelling framework}

Recall that an estimate of the EWS is provided by the square of the empirical wavelet coefficients under a NDWT (Equation 2). We consider modelling the raw wavelet periodogram across 
location $k$ over the different scales $j$. We adopt the convention that $j=1$ is the finest scale, and $j=2, \ldots, J$ as the subsequent coarser scales (where $J=\left\lfloor\log _{2} n\right\rfloor$ ). Within-scale dependence induced by the NDWT can be accounted for by the HMM framework. We refer to the collection of $J$ periodogram coefficients at a particular time point as $\mathbf{I}_{k}=\left\{I_{j, k}\right\}_{j=1, \ldots, J}$ (random variable) and $\mathbf{d}_{k}^{2}=\left\{d_{j, k}^{2}\right\}_{j=1, \ldots, J}$ (observed, empirical) from here onwards. We next turn to deriving the joint density of $\mathbf{I}_{k}$.

\subsubsection{Distribution of $\mathbf{I}_{k}$}

Recall that since we have assumed an LSW model and Gaussian innovations, $Y_{t}$ is Gaussian with mean zero. By performing a wavelet transform, the wavelet coefficients $D_{j, k}$ are Gaussian distributed themselves with mean zero. As is well documented in the literature (see Nason et al. (2000)), the use of NDWT induces a dependence structure between neighbouring $D_{j, k}$. We consider in particular, $\mathbf{D}_{k}=\left\{D_{j, k}\right\}_{j=1, \ldots, J}$, the coefficients across $J$ scales considered at a given location, $k$. Thus,

$$
\mathbf{D}_{k} \sim \operatorname{MVN}\left(\mathbf{0}, \Sigma_{k}^{D}\right) \quad k=1, \ldots, n,
$$

where $\Sigma_{k}^{D}$ specifies the covariance structure between the wavelet coefficients at location $k$ across the $J$ scales. Section 3.1.2 discusses how $\Sigma_{k}^{D}$ can be computed from the spectrum $W_{j}^{2}\left(\frac{k}{n}\right)$.

As $\mathbf{I}_{k}=\mathbf{D}_{k}^{2}=\left(D_{1, k}^{2}, \ldots, D_{J, k}^{2}\right)$, the following result can be established.

Proposition 1. The density of $\mathbf{I}_{k}$ is,

$$
\begin{aligned}
g\left(\mathbf{d}_{k}^{2} \mid \Sigma_{k}^{D}\right) & =g\left(d_{1, k}^{2}, \ldots, d_{J, k}^{2} \mid \Sigma_{k}^{D}\right) \\
& =\frac{1}{2^{J} \prod_{j=1}^{J}\left|d_{j, k}\right|} \sum_{a_{1}, \ldots, a_{J}=\{+,-\}} f\left(a_{1}\left|d_{1, k}\right|, \ldots, a_{J}\left|d_{J, k}\right| \mid \mathbf{0}, \Sigma_{k}^{D}\right),
\end{aligned}
$$

where $f\left(\cdot \mid \mathbf{0}, \Sigma_{k}^{D}\right)$ is the joint density corresponding to $\operatorname{MVN}\left(\mathbf{0}, \Sigma_{k}^{D}\right)$.

Proof. This is based on a change of variables argument detailed further in Section 1 of Supplementary Material.

We can thus use the joint density of wavelet coefficients, $\mathbf{D}_{k}$, to deduce the joint density for the squared wavelet coefficients $\mathbf{I}_{k}=\mathbf{D}_{k}^{2}$. A similar joint density can be computed if we consider each scale process of the periodogram, $\mathbf{I}^{j}=\left\{I_{j, k}\right\}_{k=1}^{n}$, although the order of computation increases exponentially. 


\subsubsection{Computing $\Sigma_{k}^{D}$}

We next turn to the problem of accounting for the dependence between the coefficients, induced by a NDWT, which feeds into the joint densities of $\mathbf{D}_{k}$ and $\mathbf{I}_{k}$. Recall that the EWS characterises the autocovariance structure of the observation process for any orthonormal incremental process as follows (Nason et al., 2000):

$$
\operatorname{Cov}\left(Y_{t}, Y_{t-v}\right)=\sum_{l} \sum_{m} W_{l}^{2}\left(\frac{m}{n}\right) \psi_{l, m}(t) \psi_{l, m}(t-v)
$$

It is possible to compute this autocovariance quantity without knowing the entire EWS due to the compact support of wavelets.

As the following proposition demonstrates, the autocovariance structure of the observations also feeds into the covariance structure of the wavelet coefficients.

Proposition 2. For a LSW process, the covariance structure between specific wavelet coefficients of a NDWT, $D_{j, k}$ is of the following form:

$$
\operatorname{Cov}\left(D_{j, k}, D_{j^{\prime}, k^{\prime}}\right)=\sum_{t} \sum_{v} \psi_{j, k}(t) \psi_{j^{\prime}, k^{\prime}}(t-v) \operatorname{Cov}\left(Y_{t}, Y_{t-v}\right)
$$

Proof. See Section 2 of Supplementary Material.

We can thus deduce the covariance structure for the wavelet coefficients $\mathbf{D}_{k}, \Sigma_{k}^{D}$, from the EWS. Again, due to the compact support associated with wavelets, only a finite number of covariances in the summation are needed to evaluate this quantity. Consequently, the entire EWS does not need to be known to calculate the covariance between the wavelet coefficients.

One can show that to compute $\Sigma_{k}^{D}$, the covariance structure of the wavelet coefficients at location $k$, the power from locations $k-2\left(\mathcal{L}_{j}-1\right), \ldots, k$ for scale $j=1, \ldots, J$ needs to be recorded where $\mathcal{L}_{j}$ denotes the number of non-zero filter elements in the wavelet at scale $j$ (see Section 3).

\subsubsection{The HMM framework}

Having derived a joint density for the wavelet periodogram, we now turn our attention to the question of how this can be incorporated appropriately within a HMM framework. The $J$ multivariate scale processes from a raw wavelet periodogram can be modelled simultaneously via a single HMM framework with the derived multivariate emission density. That is, at location 
$k$, we consider $\mathbf{I}_{k}=\left\{I_{j, k}\right\}_{j=1, \ldots, J}$, and model it as being dependent on a single underlying, unobserved Markov chain (MC), $X_{k}$, which takes values from $\Omega_{X}=\{1, \ldots, H\}$ with $H=$ $\left|\Omega_{X}\right|<\infty$

$$
\begin{aligned}
p\left(x_{k} \mid x_{1: k-1}, \theta\right) & =p\left(x_{k} \mid x_{k-1}, \theta\right) & k=1, \ldots, n & \text { (Transition) } \\
\mathbf{I}_{k} \mid\left\{X_{1: k-1}, \mathbf{I}_{1: k-1}=\mathbf{d}_{1: k-1}^{2}\right\} & \sim g\left(\mathbf{I}_{k}=\mathbf{d}_{k}^{2} \mid x_{k-2\left(\mathcal{L}_{J}-1\right): k}, \theta\right) & k=1, \ldots, n & \text { (Emission) }
\end{aligned}
$$

The HMM framework assumes that the emission density of $\mathbf{I}_{k}$ is determined by the latent process $X_{k}$, such that the process follows the Markov property and the $\mathbf{I}_{1: n}$ are conditionally independent given $X_{1: n}$. This latter remark allows us to account for the within-scale dependence induced by a NDWT. $H$ denotes the number of underlying states the latent $\mathrm{MC}, X_{k}$, can take and corresponds to different data generating mechanisms, for example "stormy" and "nonstormy" seasons in the motivating oceanographic application. Under our setup, this corresponds to the number of unique power configurations over the disjoint partitioning $\mathcal{W}_{1}, \ldots, \mathcal{W}_{H^{*}}$. That is $H \leq H^{*}$ is the number of states that generate the $H^{*}$ partitions, with some partitions possibly being generated by the same state. We assume in our analysis that $H$ is known a priori, as we want to give a specific interpretation to the states in the application, that of "stormy" or "non- stormy"seasons. Typically, in more general time series, this is not the case, and recent methods to estimate $H$ include Robert et al. (2000), Mackay (2002), Chopin (2007) and Zhou et al. (2012). In particular, we advocate the SMC based method proposed by Nam et al. (2012a), which expands upon the SMC samplers framework outlined in this manuscript to estimate the posterior distribution for the number of states. This requires no additional cost if various parameter posteriors are being approximated, each assuming a different number of states being present. It is worth mentioning that even though the order of the HMM needs to be chosen a priori the methodology is reasonably robust to the choice of the order. Indeed, as shown in Nam et al. (2012a), model selection to choose this order can be implemented given the algorithm used in the underlying analysis proposed here. In addition, we assume that the underlying unobserved $\mathrm{MC}, X_{k}$, is first order Markov, although extensions to an $q$-th order Markov Chain are permitted via the use of embedding arguments.

The state-dependent emission density, $g\left(\mathbf{I}_{k} \mid X_{k-2\left(\mathcal{L}_{J}-1\right): k}\right)$, is that proposed in Equation 4, with the covariance structure $\Sigma_{k}^{D}$ being dependent on $X_{k-2\left(\mathcal{L}_{J}-1\right): k}$. Rather than estimating entries of $\Sigma_{k}^{D}$ directly, we instead estimate the powers, $w_{j, r}^{2}$ as in Equation 3, that feed directly 
into and populate $\Sigma_{k}^{D}$. More specifically, we estimate state-dependent powers $w_{j, r}^{2}$ in

$$
W_{j, X_{k}}^{2}\left(\frac{k}{n}\right)=\sum_{r=1}^{H} w_{j, r}^{2} \mathbf{1}_{\left[X_{k}=r\right]} \quad j=1, \ldots, J .
$$

This state-dependent power structure is equivalent to the piecewise constant EWS as in Equation 3. As $X_{k}$ is permitted to move freely between all states of $\Omega_{X}$, we are able to reduce the summation limit in Equation 3 to $H$ from $H^{*}$. Returning to previous power configurations in the EWS is therefore possible, with a change in state corresponding to a change in power in at least one scale. $\Sigma_{k}^{D}$ is dependent on the underlying states of $X_{k}$ from times $k-2\left(\mathcal{L}_{J}-1\right), \ldots, k$ (see Section 3) and thus the order of the HMM is $2 \mathcal{L}_{J}-1$.

Here, $\theta$ denotes the model parameters that need to be estimated which consists of the transition matrix $\mathbf{P}$ and the aforementioned state-dependent power $W^{2}=\left\{W_{\cdot, 1}^{2}, \ldots, W_{\cdot, H}^{2}\right\}$, where $W_{\cdot, r}^{2}=\left\{w_{j, r}^{2}\right\}_{j=1}^{J}$ for all $r \in \Omega_{X}$, is associated with the emission density. Transition parameters are also of interest in their own right as they also provide information into the long run occurrence rates of changepoints, via standard Markov chain arguments.

We can partition the model parameters into transition and emission parameters, $\theta=$ $\left(\mathbf{P}, W^{2}\right)$. As $\theta$ is unknown, we turn to SMC samplers (Del Moral et al., 2006) for their estimation. As in many applications including the oceanographic one here, it is likely that data within segment types (stormy or non-stormy) are similar. The advantage of using an HMM approach is that estimation of segmental parameters then uses the combined information across all data classified as being of the same type. Section 3.2 outlines an example implementation in approximating the posterior of $\theta, p\left(\theta \mid \mathbf{d}_{1: n}^{2}\right)$.

\subsection{SMC samplers implementation}

This section outlines an example SMC implementation in approximating the parameter posterior, $p\left(\theta \mid \mathbf{d}_{1: n}^{2}, H\right)$ via a weighted cloud of $N$ particles, $\left\{\theta^{i}, U^{i} \mid H\right\}_{i=1}^{N}$, since $\theta=\left(\mathbf{P}, W^{2}\right)$ is unknown. $\theta^{i}$ indicates a potential parameter particle (a configuration of $\theta$ ) with associated weighting $U^{i}$. SMC samplers provide an algorithm to sample from a sequence of connected distributions via importance sampling and resampling techniques (Del Moral et al., 2006). Defining $l\left(\mathbf{d}_{1: n}^{2} \mid \theta, H\right)$ as the likelihood, and $p(\theta \mid H)$ as the prior of the model parameters, we can define 
the following sequence of distributions,

$$
\pi_{b}(\theta) \propto l\left(\mathbf{d}_{1: n}^{2} \mid \theta, H\right)^{\gamma_{b}} p(\theta \mid H) \quad b=1, \ldots, B
$$

where $\left\{\gamma_{b}\right\}_{b=1}^{B}$ is a non-decreasing tempering schedule such that $\gamma_{1}=0$ and $\gamma_{B}=1$. The sequence of distributions thus introduces the effect of the likelihood gradually such that we eventually sample from the parameter posterior of interest. We sample initially from $\pi_{1}(\theta)=p(\theta \mid H)$ either directly or via importance sampling, and continually mutate and reweight existing samples from the current distribution to sample the next distribution in the sequence. Resampling occasionally occurs to maintain stability in the approximation. Under the HMM framework, this does not require sampling the underlying state sequence due to the exact computation of the likelihood via the Forward-Backward Equations (Baum et al., 1970), which leads to a reduction in Monte Carlo sampling error.

Section 5 provides a detailed outline of the example SMC implementation used within our framework. The general points to note are that we consider the transition probability row vectors, $p_{r}, r=1, \ldots, H$ constructing transition matrix $\mathbf{P}$ independently from the inverse state dependent powers $\frac{1}{w_{j, r}^{2}}, j=1, \ldots, J, r=1, \ldots, H$. The re-parametrisation of the state dependent powers to its inverse is analogous to the re-parametrisation of variance to precision (inverse variance) in typical time-domain models. In practice, the series we consider will all contain at least a small portion of variation, and as such issues regarding zero or infinite power for particular frequencies will not arise. We initialise by sampling from a Dirichlet and Gamma prior distribution respectively for transition probability vectors and state dependent powers, and mutate according to a Random Walk Metropolis Hastings Markov kernel on the appropriate domain for each component. There is a great deal of flexibility within the SMC samplers framework with regards to the type of mutation and sampling schemes from the prior. The example implementation presented is in no way the only implementation or optimal with respect to optimising mixing and acceptance rates. However, this design provides results which appear sensible without a great deal of manual tuning.

\subsection{Exact changepoint distributions}

Having formulated an appropriate HMM framework to model the periodogram $\mathbf{d}_{1: n}^{2}$, and accounting for unknown $\theta$ via SMC samplers, it is now possible to compute the changepoint 
distributions of interest. Conditioned on $\theta$, exact changepoint distributions, such as $P\left(\tau^{\left(k_{\mathrm{CP}}\right)}=\right.$ $\left.t \mid \mathbf{d}_{1: n}^{2}, \theta, H\right)$, can be computed via Finite Markov Chain Imbedding (FMCI) in a HMM framework (see Aston et al. (2011) and references therein). The exact nature refers to the fact that the distributions are not subject to sampling or approximation error, conditioned on $\theta$. The FMCI framework uses a generalised changepoint definition such that sustained changes in state are permitted, which corresponds to the sustained nature of seasons lasting at least a few time periods. The sustained nature is captured via the following definition,

Definition 2. A changepoint to a regime occurs at time $t$ when a change in state persists for at least $k_{\mathrm{CP}}$ time periods. That is

$$
X_{t-1} \neq X_{t}=\ldots=X_{t+j}
$$

where $j \geq k_{\mathrm{CP}}-1$.

We say a changepoint-out-of the regime corresponding to state $s \in \Omega_{X}$ is said to have occurred at time $t^{\prime}$ when $X_{t}$ has not been in state $s$ for $k_{\mathrm{CP}}^{\prime}$ time periods. That is

$$
X_{t^{\prime}-j} \neq s \quad \forall 0 \leq j \leq k_{\mathrm{CP}}^{\prime}-1 .
$$

This definition generalises the changepoint-out-of the regime $k_{\mathrm{CP}}^{\prime}=1$, considered in Aston et al. (2011). Under this changepoint definition, the changepoint problem becomes a waiting time distribution problem for runs in the underlying state sequence. The framework is flexible and efficient such that a variety of distributions regarding changepoint characteristics can be computed. In particular, probabilities can be calculated for changes to have occurred within a region rather than at a specific point. By considering the probability of a changepoint within an interval around a location, one can obtain probabilistic statements about the existence of a changepoint occurring within a region. Comparing across regions obtained, one can thus identify the most probable regions of change.

\subsection{Outline of Approach}

An outline of the final algorithm is as follows:

1. Perform a NDWT to time series $y_{1: n}, n=2^{J}, J \in \mathbb{N}$ to obtain the wavelet periodogram.

2. Let $\mathbf{d}_{1: n}^{2}$ denote the corresponding $J$ multivariate time series from the periodogram. 
3. Assuming $H$ underlying states, model $\mathbf{d}_{1: n}^{2}$ by a HMM framework with the corresponding joint emission density. This density accounts for the dependence structure between scale processes.

4. Account for the uncertainty of the unknown HMM model parameters, $\theta=\left(\mathbf{P}, W^{2}\right)$, via Sequential Monte Carlo samplers. This results in approximating the posterior, $p\left(\theta \mid \mathbf{d}_{1: n}^{2}, H\right)$, by a weighted cloud of $N$ particles $\left\{\theta^{i}, U^{i} \mid H\right\}_{i=1}^{N}$.

5. To obtain the changepoint probability of interest, approximate as follows. Let $k_{\mathrm{CP}}$ denotes the sustained condition under a generalised changepoint definition (Nam et al., 2012b), and $P\left(\tau^{\left(k_{\mathrm{CP}}\right)}=t \mid \mathbf{d}_{1: n}^{2}, \theta^{i}, H\right)$ to be the exact changepoint distribution conditional on $\theta^{i}$. Then the changepoint probability is,

$$
P\left(\tau=t \mid y_{1: n}\right) \equiv P\left(\tau^{\left(k_{\mathrm{CP}}\right)}=t \mid \mathbf{d}_{1: n}^{2}, H\right) \approx \sum_{i=1}^{N} U^{i} P\left(\tau^{\left(k_{\mathrm{CP}}\right)}=t \mid \mathbf{d}_{1: n}^{2}, \theta^{i}, H\right) .
$$

That is, the weighted average of conditional exact changepoint distributions with respect to different model parameter configurations. $P\left(N_{C P}=n_{C P} \mid y_{1: n}\right) \equiv P\left(N_{C P}^{\left(k_{\mathrm{CP}}\right)}=\right.$ $\left.n_{C P} \mid \mathbf{d}_{1: n}^{2}, H\right)$ follows analogously.

Computationally, it is not possible to consider all $J$ scales of the periodogram as the order of the HMM increases exponentially (see Section 4 for further details). Consequently, we approximate by considering $J^{*} \leq J$ finer scales of the periodogram, a common approach in time series analysis (see for example Cho and Fryzlewicz (2012)). This restricts our attention to changes in autocovariance structure associated at higher frequencies which seem more apparent in the oceanographic data of interest. This should therefore not hinder our proposed methodology with regards to the motivating application.

We assume that the choice of analysing wavelet used for the transform is known a priori, and is the same as the generating wavelet. However, this is often unknown and we note that wavelet choice is an area of ongoing interest with the effect between differing generating and analysing wavelets for EWS estimation investigated in Gott and Eckley (2013). 


\section{Results and Applications}

We next consider the performance of our proposed methodology on both simulated and oceanographic data.

We first consider simulated white noise and MA processes with piecewise second-order structures. In particular, white noise processes are considered and compared to a time-domain HMM approach because this type of process can be modelled exactly in the time-domain. Hence our proposed wavelet method should thus compliment it. The potential benefit of the proposed wavelet approach is then demonstrated on piecewise MA processes in which an exact timedomain HMM is not possible. In addition, we compare with estimates provided by AutoPARM (Davis et al., 2006) and CF (Cho and Fryzlewicz, 2012), demonstrating that our methodology performs on a par with them and the importance of quantifying the uncertainty of changepoints.

We also return to the motivating oceanographic application. In addition to quantifying the uncertainty of the changepoints, we demonstrate concurrence with estimates provided by other autocovariance changepoint methods and those provided by expert oceanographers.

\subsection{Simulated Data}

We consider simulated processes of length 512 and with defined changepoints (purple dotted lines at times 151, 301 and 451). We compare our proposed method to a time-domain Gaussian Markov Mixture model on the time series itself, regardless of how the data is actually generated.

In generating our results, the following SMC samplers settings have been used; $N=500$ samples to approximate the defined sequence of $B=100$ distributions. The hyperparameter for the $r$-th transition probability vector, $\alpha_{r}$, is a $H$-long vector of ones with 10 in the $r$-th position which encourages the underlying MC to remain in the same state. The shape and scale hyperparameters for the inverse power parameters priors are $\alpha_{\lambda}=1$ and $\beta_{\lambda}=1$ respectively. A linear tempering schedule, that is $\gamma_{b}=\frac{b-1}{B-1}, b=1, \ldots, B$, and a baseline line proposal variance of 10 which decreases linearly with respect to the iteration of the sampler, are utilised.

We consider processes arising from two possible generating mechanisms in the time-domain, and we thus assume $H=2$ in our HMM framework, and $k_{\mathrm{CP}}=20, k_{\mathrm{CP}}^{\prime}=10$ for the required sustained change in state under our changepoint definition. $J^{*}=3$ scale processes of the periodogram under a Haar LSW framework are considered, a computationally efficient setting 
under the conditions presented.

For the SMC implementation regarding the Gaussian Markov Mixture model, the following priors were implemented: $\mu_{r} \stackrel{\mathrm{iid}}{\sim} \mathrm{N}(0,10), \frac{1}{\sigma_{r}^{2}} \stackrel{\mathrm{iid}}{\sim}$ Gamma(shape $=1$, scale $\left.=1\right), r=1,2$.

\subsubsection{Gaussian White Noise Processes with Switches in Variance}

The following experiment concerns independent Gaussian data which exhibits a change in variance at defined time points. It is well known that the corresponding true EWS is $W_{j}^{2}\left(\frac{k}{n}\right)=$ $\frac{\sigma_{k}^{2}}{2^{j}}, j=1, \ldots, J$. A change in variance thus causes a change in power across all scales simultaneously. No approximation is required under a time-domain approach, as this change can be represented exactly. A single realisation of the data and corresponding changepoint analysis are displayed in Figure 2. The top panel is a plot of the simulated data analysed with changepoint estimates provided by $\mathrm{CF}$ and AutoPARM denoted by the top blue and bottom red ticks respectively. The second and third panel display the changepoint probability plot (CPP, the probability of a changepoint occurring at each time) under the wavelet and time-domain approaches respectively. The fourth panel presents the distribution of the number of changepoints from both approaches.

We observe that our proposed methodology has peaked and centred CPP around the defined changepoint locations and provides similar results to the time-domain approach. This type of $\mathrm{CPP}$ behaviour provides an indication of the changepoint location estimates. In some instances, the wavelet approach outperforms the time-domain approach, for example the changepoint associated with time point 301 is more certain. We note that there is some significant CPP assigned to the first few time points under the wavelet approach. This arises due to a label identifiability issue common with HMMs (see Scott (2002)), and the fact that the initialisation associated with these labels was designed with the oceanographic application in mind, where the initial storm season status is known. However, should this not be the case in another application, a diffuse initialisation across all states could be used. However, under the known initialisation setting, an additional changepoint is often detected at the start of the data in the simulations and this is reflected in the changepoint distribution. Disregarding this artefact, we observe that three changepoints occurring is almost certain under the wavelet approach. This is in accordance with the time-domain approach and truth. 
[Figure 2 about here.]

The results demonstrate that there is potential in providing an alternative method when dealing with this type of data as the wavelet based method identifies changepoints near the defined locations. However some differences and discrepancies do exist between the proposed wavelet approach, the truth and time-domain approach. In particular, the CPP under the proposed approach is slightly offset from the truth. However, these estimates are still in line with what we might observe in the time series realisation and compares favourably to the time-domain approach and existing approaches.

\subsubsection{Piecewise MA processes - Piecewise Haar MA processes}

The following scenario considers piecewise MA processes with changing MA order. We consider in particular piecewise Haar MA processes where the coefficients of the MA process are the Haar wavelet coefficients with a piecewise constant power structure in the EWS being present. Such processes are the types of data that our proposed methodology should perform well on and for which time-domain HMM methods require some approximation involving high (but arbitrary and fixed) AR orders. In this case, we model the observed time series as a Gaussian Markov Mixture in the time-domain (AR order is zero). This incorrect modelling approach is also equally applicable when dealing with real data where the "true" model is unknown.

Stationary Haar MA processes have constant power structure in a single scale $j^{\prime}$ of the EWS, namely $W_{j}^{2}\left(\frac{k}{n}\right)=\mathbf{1}_{\left[j=j^{\prime}\right]} \sigma^{2}, \quad j^{\prime} \in\{1, \ldots, J\}$, and a Haar generating wavelet, where $\sigma^{2}$ is the time-domain innovation variance of the process. The equivalent time-domain representation of this model is a $\operatorname{MA}\left(2^{j^{\prime}}-1\right)$ process with innovation variance $\sigma^{2}$ and MA coefficients determined by the Haar wavelet at scale $j^{\prime}$. Piecewise Haar MA processes can thus be constructed by considering piecewise constant EWS. Changes in power across scales correspond to changes in MA order and changes in power within-scales correspond to changes in variance of $Y_{t}$. Nason et al. (2000) remark that any MA process can be written as a linear combination of Haar MA processes, with the wavelet representation often being sparse.

Figure 3 considers a single realisation of a change in order from $\operatorname{MA}(1) \leftrightarrow \operatorname{MA}(7)$ and constant variance $\sigma^{2}=1$. These results show the real potential of the proposed method in that the CPP are centred and peaked around the defined changepoint locations, with addi- 
tional changepoint potentially being present. The potential presence of additional changepoints (more correctly, models where including additional changepoints allows an improved fit to this particular finite sample data series over the true data generating process) is also reflected in the distribution of the number of changepoints with probability assigned to these number of changepoints. This reflects the nature of individual simulations, where the number of changes varies dependent on the realisation. However, given the nature of the HMM, estimation of the parameters is still across all segments rather than purely within any one individual segment. In contrast to the wavelet approach, the time-domain method is unable to identify changepoints due to the highly correlated nature and change of autocovariance present in the the data. This thus demonstrates that there is an advantage in considering the changepoint problem in the wavelet-domain over the time-domain, in light of incorrect model specification. Our method also compares favourably with existing autocovariance changepoint methods.

[Figure 3 about here.]

Further piecewise MA simulations were performed with regards to changing variance, and both changing variance and MA order simultaneously (results not shown here). Under such scenarios, the proposed methodology outperformed or compared favourably to the approximating time-domain approach.

[Figure 4 about here.]

In assessing the performance of our methodology, Figure 4 displays summaries of the CPP across 50 simulations for the two types of data considered. We observe that the proposed methodology works well with the general CPP being peaked and centred around the defined CP locations with no other possible changes detected. The small offset is due to the fact that we are averaging skewed distributions, the skew arising from the fact that we insist on sustained changes for detection, as is required in the Oceanographic example.

\subsection{Oceanographic Application}

We now return to consider the oceanographic data example introduced in Section 1. Clearly there is ambiguity as to when storm seasons start and the number that have occurred. Hence 
there is particular interest in quantifying the uncertainty of storm seasons. We therefore apply our proposed methodology to the data from a location in the North Sea.

The analysed data is plotted in the top panel of Figure 5 along with changepoint estimates from existing change in autocovariance methods namely, Cho and Fryzlewicz (2012) (CF, top (blue) ticks) and Davis et al. (2006) (AutoPARM, bottom (red) ticks). The data consists of differenced wave heights measured at 12 hour intervals from March 1992 - December 1994 in a central North Sea location.

The following inputs have been used to achieve the presented changepoint results in Figure 5: $J^{*}=2$ corresponding to higher frequency time series behaviour (where changes are expected), and $H=2$ states have been assumed reflecting the belief that there are "stormy" and "nonstormy" seasons. The same SMC samplers settings utilised in the simulated data analysis have been used ( $N=500$ particles, $B=100$ distributions, linear tempering schedule). Under a sustained changepoint definition, $k_{\mathrm{CP}}=40$ and $k_{\mathrm{CP}}^{\prime}=30$, have been used to reflect the general sustained nature of seasons (seasons last for at least a few weeks). The initialisation reflected the fact that the storm status of the data was known at the start.

Ocean engineers have indicated that it is typical to see two changes in storm season each year occurring in the Spring (March-April) and Autumn (September-October). The results displayed in Figure 5 concur with this statement; five and six storm season changes are most likely according to the number of changepoints distribution, and with the CPP being centred and peaked around these times. The uncertainty encapsulated by the number of changepoint distribution demonstrates that there are potentially more or fewer storm seasons than five or six, although these are less certain, along with the corresponding locations.

Results also concur with changepoint estimates from the other two methods, with our method highlighting another possible configuration. A few discrepancies exist, for example the changepoint estimated in the middle of 1993 according to CF and AutoPARM. These potential changes in state do not seem sufficiently sustained for a change in season to have occurred and thus our methodology has not identified them. Lowering the associated values of $k_{\mathrm{CP}}$ and $k_{\mathrm{CP}}^{\prime}$, does begin to identify these as changepoint instances, in addition to others. Changes identified in the middle of 1992 and end of 1994 by CF and AutoPARM are suspected to be due to an insufficient number of states to account for these more subtle changes. HMM model selection 
methods may therefore be worth implementing, although the current two state assumption corresponds directly to "stormy" and "non-stormy" seasons, allowing the model to be easily interpreted by ocean engineers.

[Figure 5 about here.]

\section{Discussion}

This paper has proposed a methodology for quantifying the uncertainty of autocovariance changepoints in time series. This is achieved by considering the estimate of the Evolutionary Wavelet Spectrum which fully characterises the potentially varying second-order structure of a time series. By appropriately modelling this estimate as a multivariate time series under a Hidden Markov Model framework and deriving the corresponding multivariate emission density which accounts for the dependence structure between processes, we can quantify the uncertainty of changepoints. The uncertainty of autocovariance changepoints has not explicitly been considered by existing methods in the literature.

While in this paper we have concentrated on quantification of uncertainty, it should be noted that HMMs are widely used for changepoint detection, and if specific changepoint locations are required, then any number of algorithms based on maximum a-posteriori or other criteria can be used to make these choices. However, these locations will be subject to the inherent uncertainty as demonstrated by the uncertainty found by the presented approach.

This methodology has been motivated by oceanographic data exhibiting changes in secondorder structure (corresponding to changes in storm season) where there is interest in the uncertainty of storm season changes due to their inherent ambiguity. Our method has showed accordance with various existing changepoint methods including expert ocean engineers. Our methodology allows us to assess the plausibility and performance of changepoint estimates and provide further information in planning future operations. A few discrepancies do exist between the various methods, a potential result of the sustained changepoint definition implemented and number of states assumed in our HMM. However, the settings used to achieve the results seem valid given the oceanographic application and are more intuitive in controlling changepoint results compared to abstract tuning parameters and penalisation terms in other changepoint 
methods.

It might be postulated that first order regression changes are responsible for the time series observed, in which case methodology based on Jump Regression (Joo and Qiu, 2009; Qiu, 2005; Qiu and Yandell, 1998) might be a possible approach. However, when changes in regression using simpler models than jump regression were investigated (data not shown), these were found to not particularly well capture the structure of the data. In many ways, this accords with the oceanographic literature in that changes are expected in the second order structure (Killick et al., 2009) rather than the first order regression structure.

Results on a variety of simulated data also indicate that the methodology works well in quantifying the uncertainty of changepoint characteristics. Comparisons with a time-domain approach demonstrate the real advantage of our proposed methodology lies in considering piecewise MA processes which are not readily analysed using the HMM framework in the time-domain without some approximation taking place.

The current LSW framework assumes that the observed time series is mean zero and constant with prior detrending occurring before analysis is performed. However, as non-stationarity can also arise from changes in mean, future work would consider a modified version of the LSW framework such that changes in mean are also accounted for. This would thus provide a potentially powerful unified framework in which changes in mean and second-order structure are analysed simultaneously.

\section{Supplementary Material}

Technical Material: The file Nametal_tech.pdf provides further technical details for the theory and algorithms. (PDF file)

Code for Simulations: The file Nametal_code.tar.gz provides all the code and related documentation to produce the simulated examples in the paper (GNU zipped tar file)

\section{Acknowledgements}

The authors would like to thank Shell International Exploration Production for supplying the oceanographic data, and Richard Davis and Haeran Cho for providing respective codes of Au- 
toPARM and CF algorithms. Three of the authors (JA, IE, RK) would also like to acknowledge the Issac Newton Institue for Mathematical Sciences, Cambridge for support and hospitality during the programme Inference for Changepoints and Related Processes where this work was finalised. JA was partially supported in this work by EPSRC grant EP/K021672/1. RK and IE were partially supported in this work by EPSRC grant EP/I016368/1.

\section{References}

Aston, J. A. D., J. Y. Peng, and D. E. K. Martin (2011). Implied distributions in multiple change point problems. Statistics and Computing 22, 981-993.

Baum, L. E., T. Petrie, G. Soules, and N. Weiss (1970). A maximization technique occurring in the statistical analysis of probabilistic functions of Markov chains. The Annals of Mathematical Statistics 41(1), pp. 164-171.

Cappé, O., E. Moulines, and T. Rydén (2005). Inference in Hidden Markov Models. Springer Series in Statistics.

Chen, J. and A. K. Gupta (2000). Parametric Statistical Change Point Analysis. Birkhauser.

Chib, S. (1998). Estimation and comparison of multiple change-point models. Journal of Econometrics 86, 221-241.

Cho, H. and P. Fryzlewicz (2012). Multiscale and multilevel technique for consistent segmentation of nonstationary time series. Statistica Sinica 21, 671-681.

Choi, H., H. Ombao, and B. Ray (2008). Sequential change-point detection methods for nonstationary time series. Technometrics $50(1), 40-52$.

Chopin, N. (2007). Inference and model choice for sequentially ordered hidden Markov models. Journal of the Royal Statistical Society Series B 69(2), 269.

Davis, R. A., T. C. M. Lee, and G. A. Rodriguez-Yam (2006). Structural break estimation for nonstationary time series models. Journal of the American Statistical Association 101, $223-239$.

Del Moral, P., A. Doucet, and A. Jasra (2006). Sequential Monte Carlo samplers. Journal of the Royal Statistical Society Series B 68(3), 411-436.

Eckley, I., P. Fearnhead, and R. Killick (2011). Analysis of changepoint models. In D. Barber, A. Cemgil, and S. Chiappa (Eds.), Bayesian Time Series Models, pp. 215-238. Cambridge University Press.

Frühwirth-Schantter, S. (2005). Finite Mixture and Markov Switching Models. Spring Series in Statistics.

Fryzlewicz, P. and G. Nason (2006). Haar-fisz estimation of evolutionary wavelet spectra. Journal of the Royal Statistical Society: Series B (Statistical Methodology) 68(4), 611-634.

Gott, A. and I. Eckley (2013). A note on the effect of wavelet choice on the estimation of the evolutionary wavelet spectrum. Communications in Statistics-Simulation and Computation 42, 393-406. 
Joo, J. and P. Qiu (2009). Jump detection in a regression curve and its derivative. Technometrics $51,289-305$.

Killick, R., I. Eckley, K. Ewans, and P. Jonathan (2009). Detection of changes in the characteristics of oceanographic time-series using changepoint analysis. Ocean Engineering 37, $1120-1126$.

Killick, R., I. Eckley, and P. Jonathan (2013). A wavelet-based approach for detecting changes in second order structure within nonstationary time series. Electronic Journal of Statistics 7, $1167-1183$.

MacDonald, I. L. and W. Zucchini (1997). Monographs on Statistics and Applied Probability 70: Hidden Markov and Other Models for Discrete-valued Time Series. Chapman \& Hall/CRC.

Mackay, R. (2002). Estimating the order of a hidden Markov model. Canadian Journal of Statistics 30(4), 573-589.

Nam, C. F. H., J. A. D. Aston, and A. M. Johansen (2012a). Parallel Sequential Monte Carlo samplers and estimation of the number of states in a Hidden Markov model. CRiSM Research Report 12-23.

Nam, C. F. H., J. A. D. Aston, and A. M. Johansen (2012b). Quantifying the uncertainty in change points. Journal of Time Series Analysis 33(5), 807-823.

Nason, G., R. Von Sachs, and G. Kroisandt (2000). Wavelet processes and adaptive estimation of the evolutionary wavelet spectrum. Journal of the Royal Statistical Society: Series B 62(2), $271-292$.

Nason, G. P. (2008). Wavelet Methods in Statistics with R. Springer Verlang.

Percival, D. B. and A. T. Walden (2007). Wavelet Methods for Time Series Analysis. Cambridge Series in Statistical and Probabilistic Mathematics.

Qiu, P. (2005). Image Processing and Jump Regression Analysis. New York: John Wiley \& Sons.

Qiu, P. and B. Yandell (1998). A local polynomial jump detection algorithm in nonparametric regression. Technometrics 40, 141-152.

Robert, C. P., T. Rydén, and D. M. Titterington (2000). Bayesian inference in hidden Markov models through the reversible jump Markov chain Monte Carlo method. Journal of the Royal Statistical Society: Series B (Statistical Methodology) 62(1), 57-75.

Scott, S. (2002). Bayesian methods for hidden Markov models: Recursive computing in the 21st century. Journal of the American Statistical Association 97(457), 337-351.

Vidakovic, B. (1999). Statistical Modeling by Wavelets. Wiley Series in Probability and Statistics.

Viterbi, A. (1967, April). Error bounds for convolutional codes and an asymptotically optimum decoding algorithm. Information Theory, IEEE Transactions on 13(2), 260 - 269.

Wang, Y. (1995). Jump and sharp cusp detection by wavelets. Biometrika 82(2), 385-397. 
Whitcher, B., P. Guttorp, and D. Percival (2000). Multiscale detection and location of multiple variance changes in the presence of long memory. Journal of Statistical Computation and Simulation 68(1), 65-87.

Zhou, Y., A. Johansen, and J. Aston (2012). Bayesian model comparison via path-sampling sequential Monte Carlo. In Statistical Signal Processing Workshop (SSP), 2012 IEEE, pp. 245-248. IEEE. 


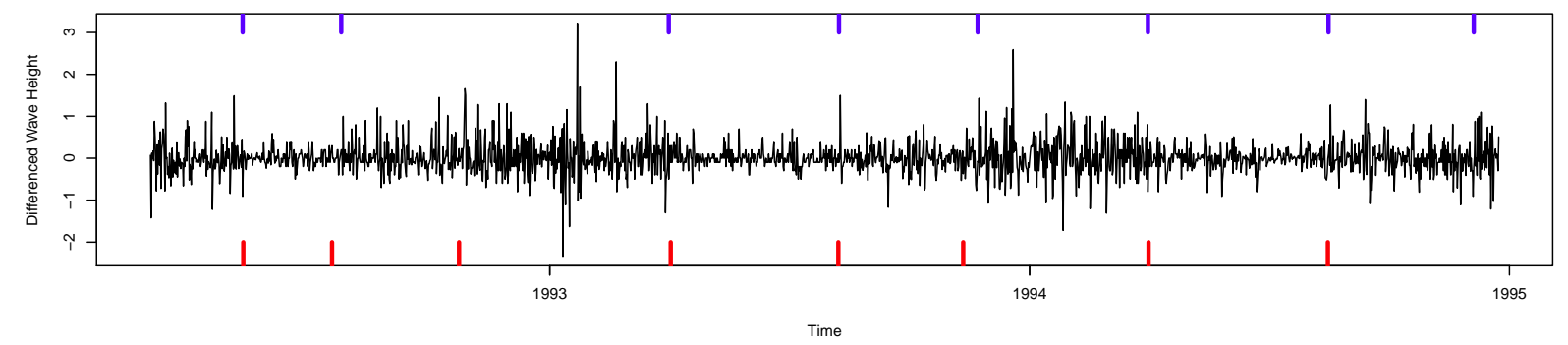

Figure 1: Changepoint estimates on wave height data from existing autocovariance changepoint methods $(\mathrm{CF}=$ top (blue) ticks, AutoPARM= bottom (red) ticks). The plot highlights that different changepoint methods will often provide different results, and thus there is a need to account for the discrepancies in methods by quantifying the uncertainty. 


\section{Simulated White Noise Data}

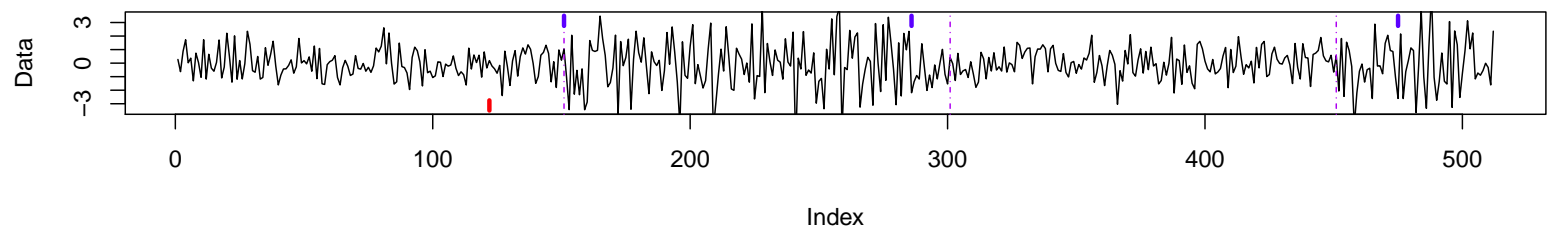

ChangePoint Probability plot (Wavelet)

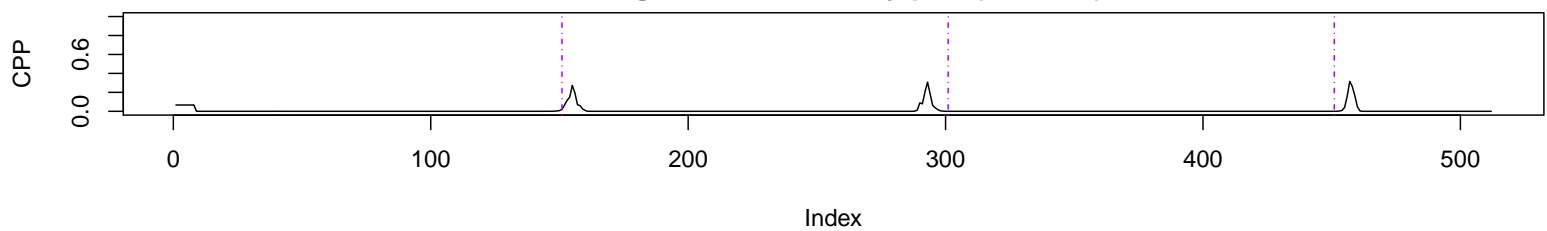

ChangePoint Probability plot (Time)
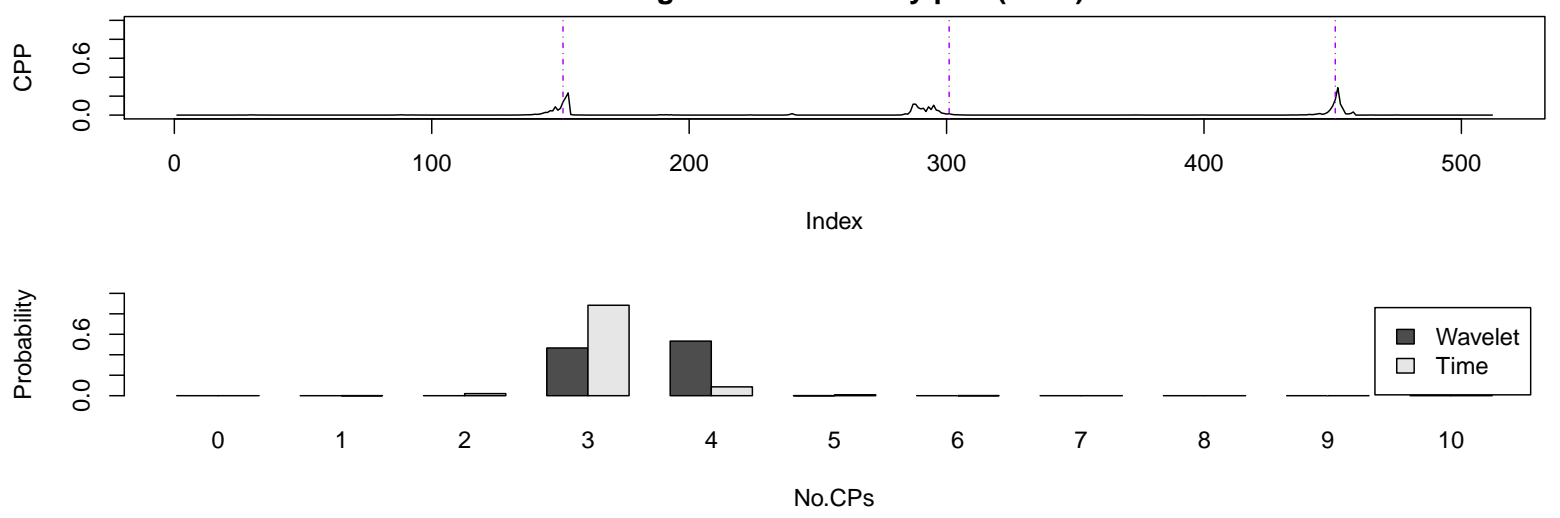

Figure 2: Changepoint results (CPP plot and distribution of number of changepoints) for simulated Gaussian white noise data with a change in variance (1 and 4). 1st panel presents the simulated data analysed. The top (blue) ticks and bottom (red) ticks denote the changepoints estimated by $\mathrm{CF}$ and AutoPARM respectively. 2nd and 3rd panel displays the CPP plots under the wavelet and time-domain approaches respectively. 4th panel presents the distribution of number of changepoints. The proposed methodology compliments the time-domain approach and concurs with the truth. 

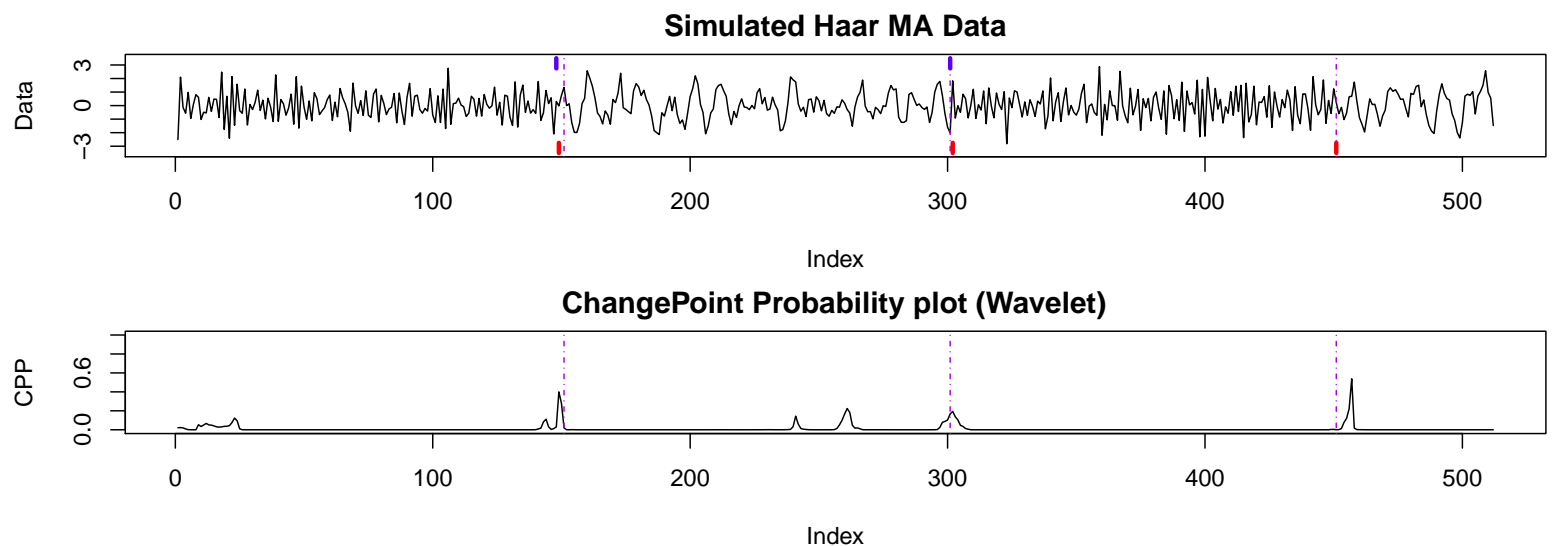

ChangePoint Probability plot (Time)
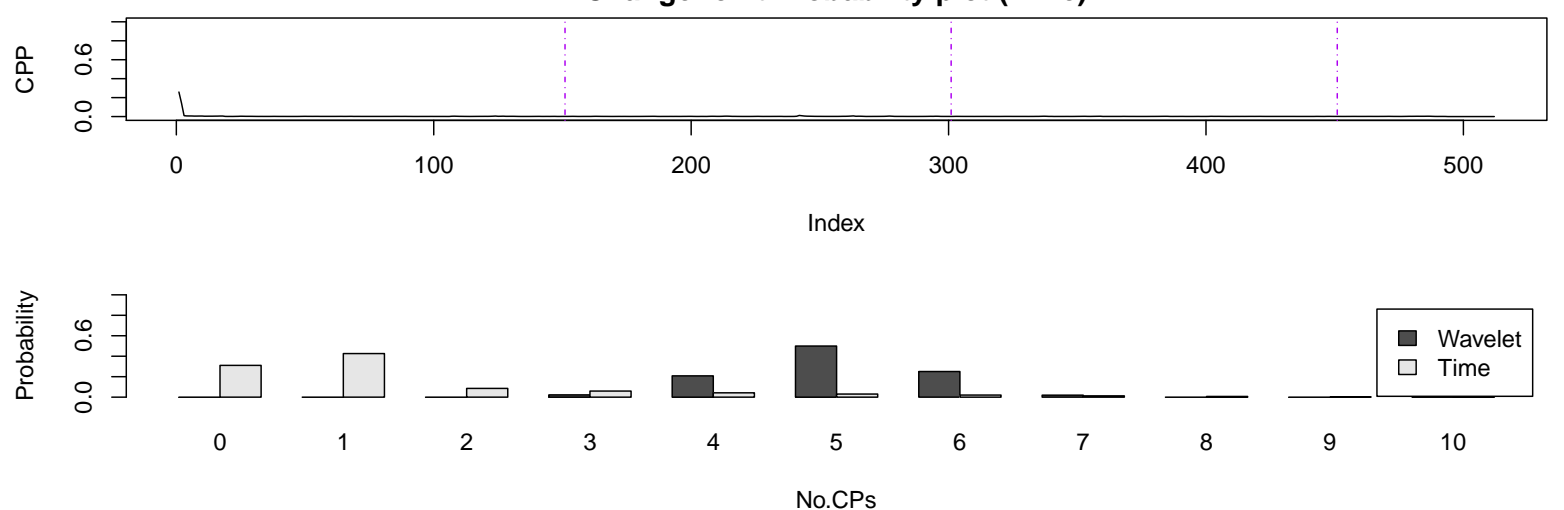

Figure 3: Changepoint results for piecewise Haar MA data with a change in order, constant variance (change in power across scales $\left.\rightarrow \mathrm{MA}(1) \leftrightarrow \mathrm{MA}(7), \sigma^{2}=1\right)$. 1st panel presents the simulated data analysed. The top (blue) ticks and bottom (red) ticks denote the changepoints estimated by $\mathrm{CF}$ and AutoPARM respectively. 2nd and 3rd panel displays the CPP plots under the wavelet and time-domain approaches respectively. 4th panel presents the distribution of number of changepoints. The wavelet-domain approach is successfully able to identify the defined changepoint locations, in addition to other changepoints. This is reflected in the distribution of the number of changepoints. The time-domain fails to identify the changepoint characteristics however due to high autocorrelation present in the data and the change within it. 


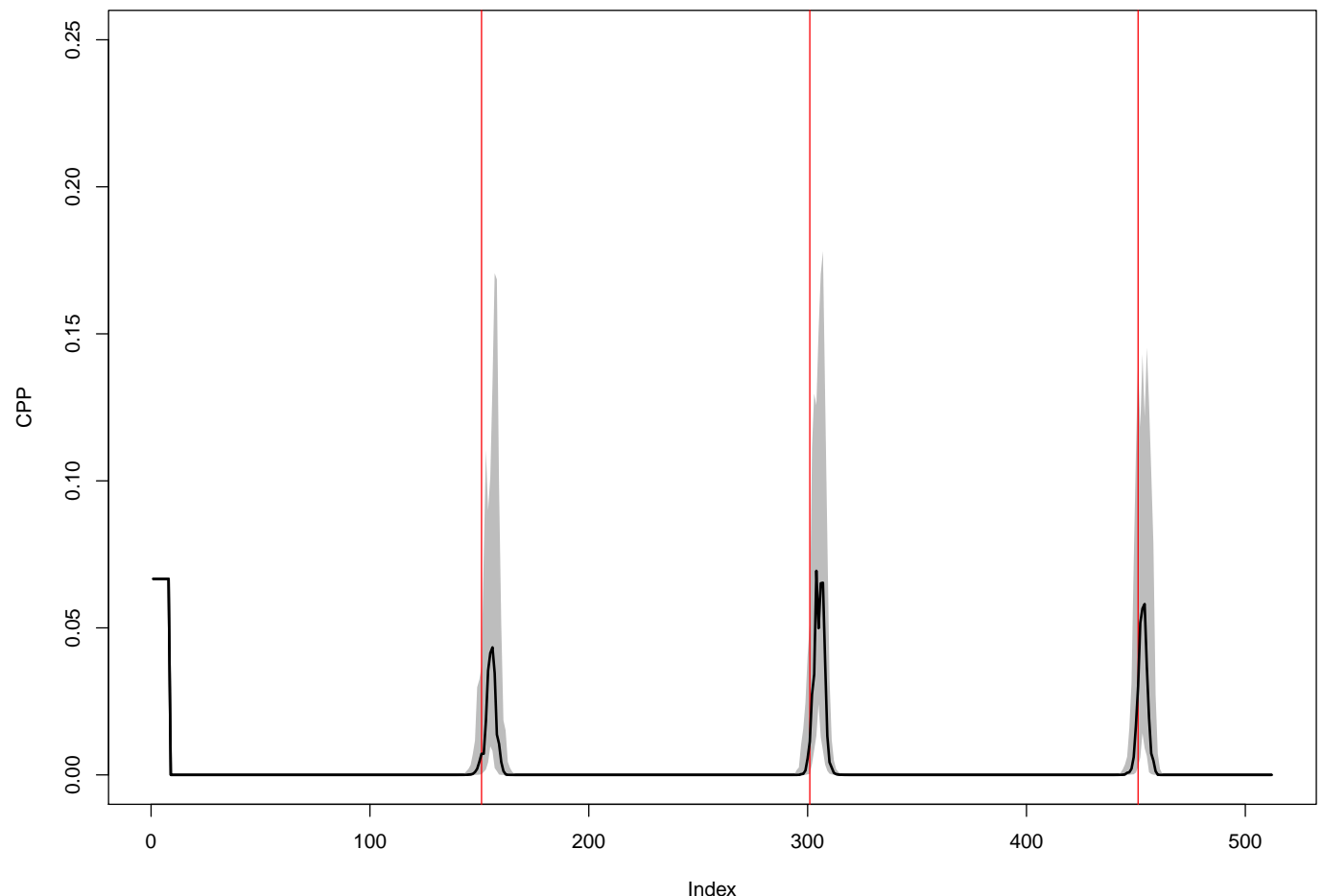

(a) White Noise data

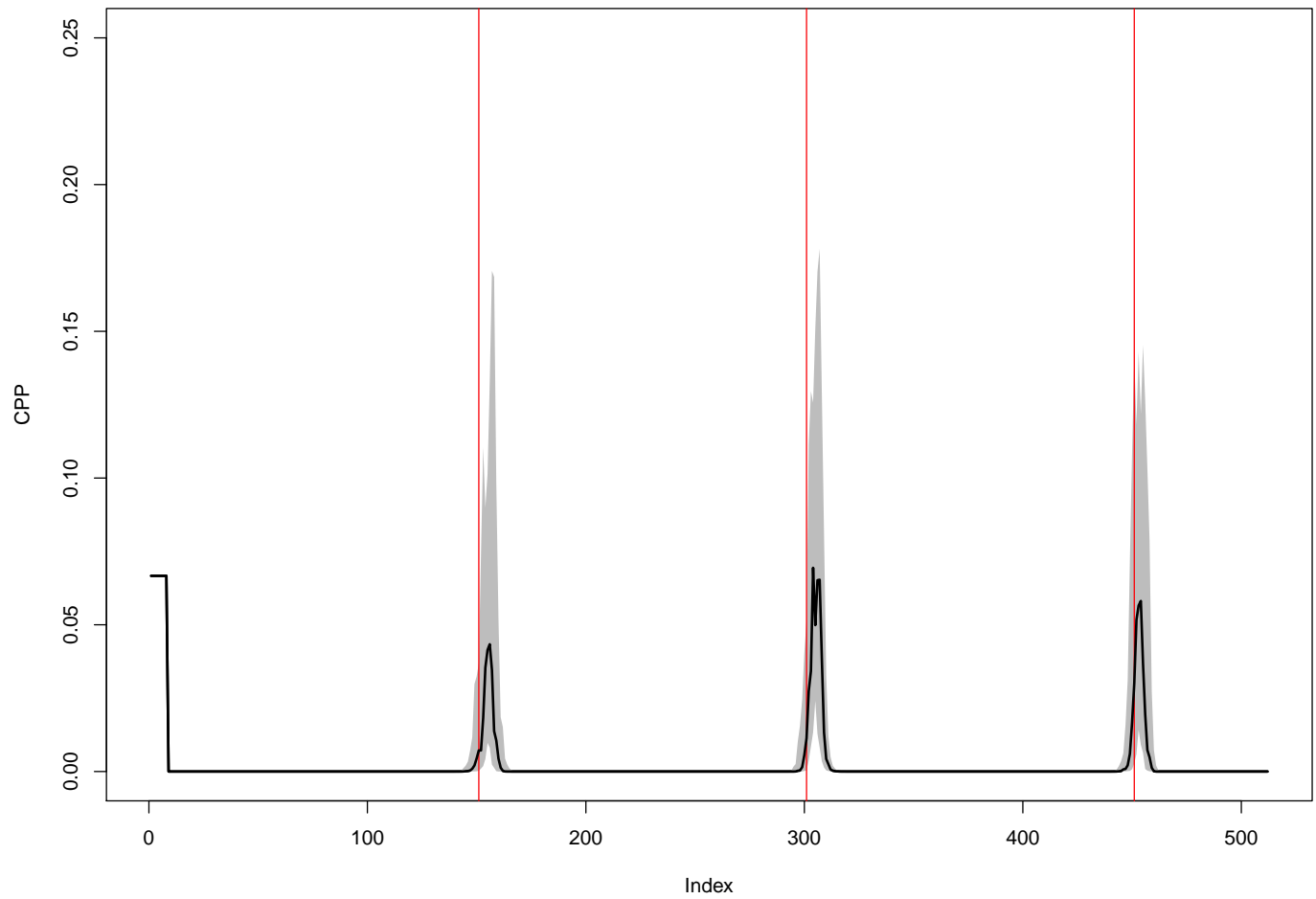

(b) HaarMA data

Figure 4: Summary of CPP from 50 simulations for the two types of data considered.. The black bold line and the grey region denote the median and interquartile range of the CPP region across 50 simulations. 


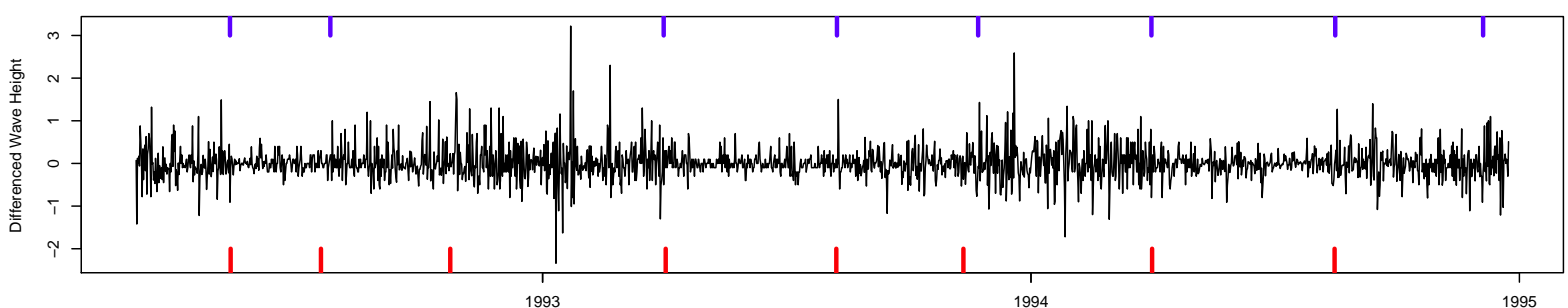

Time

ChangePoint Probability
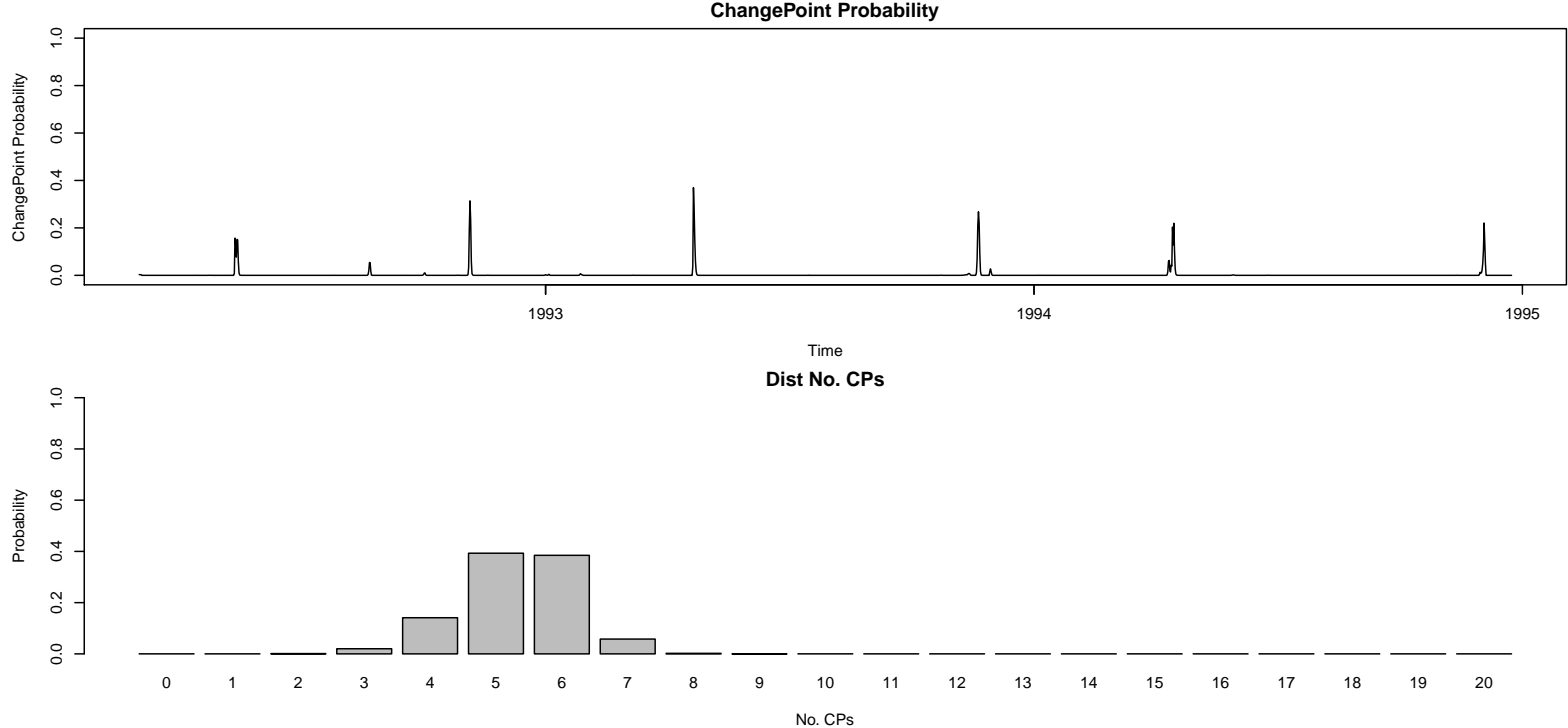

Figure 5: Changepoint results for North Sea data. Top panel displays the analysed data and the changepoint estimates from existing approaches $(\mathrm{CF}=$ top (blue) ticks, AutoPARM= bottom (red) ticks). Middle and bottom panel display the CPP plot and distribution of number of changepoints respectively under the proposed methodology. This corresponds to the start of storm seasons and the number of them. Analysis considers the two finest scales of the wavelet periodogram $\left(J^{*}=2\right)$, and assumes two underlying states $(H=2)$ reflecting "stormy" and "non-stormy" seasons. 